\title{
EFFECT OF TRYPTOPHAN RESIDUES ON ADSORPTION OF A CATIONIC ARGININE RICH POLYPEPTIDE BY HYDROXYAPATITE NANOPARTICLES AND FOLLOWING TRANSLOCATION OF THE POLYPEPTIDE THROUGH A LIPID VESICLE MEMBRANE
}

\author{
Satoru Ueno ${ }^{1, *}$, Saburo Shimabayashi ${ }^{2}$, and Hiroyuki Saito ${ }^{1}$ \\ (*Corresponding author: sueno@ph.tokushima-u.ac.jp)
}

\author{
${ }^{1}$ The University of Tokushima Institute of Health Biosciences, Graduate School Division of Physical Pharmacy \\ Sho-machi 1-78-1, Tokushima 770-8505, Japan. \\ ${ }^{2}$ Osaka University of Pharmaceutical Sciences, 4-20-1, Nasahara, Takatsuki, Osaka 569-1094, Japan.
}

Keywords: arginine rich polypeptide, tryptophan residue, hydroxyapatite, complex formation, membrane translocation

\begin{abstract}
Hydroxyapatite (abbreviated to HAp), in general, adsorbs a polypeptide, resulting in a HAp-peptide complex. In the present paper the Hap was of a nanosized particle and peptide was poly-L-arginine (abbreviated to poly(Arg)) or copoly(L-arginine/ L-tryptophan = 4:1) (abbreviated to poly(Arg,Trp)). Physicochemical properties of these complexes were quite different with each other due mainly to the presence or absence of tryptophan residues on the polymer chain. The adsorption amount of poly(Arg,Trp) on HAp was much larger than that of poly(Arg). Zeta potential of the HAp-poly(Arg,Trp) complex was far lower than that of the HAp-poly(Arg) complex. Considering these facts, it was concluded that hydrophobic interaction between Trp residues of poly(Arg,Trp) induced an adsorption bilayer on HAp, while poly(Arg) simply formed an adsorption monolayer through electrostatic interaction. On the other hand, structural flexibility of a copolymer and its translocation through a lipid membrane were lower and slower in poly(Arg, Trp) than in poly(Arg).
\end{abstract}

(Received December 30, 2010; Accepted March 29, 2011)

\section{INTRODUCTION}

Hydroxyapatite (HAp) is well known for its high biocompatibility and osteoconductivity, and is widely used as a bone substitute material ${ }^{1-3}$. HAp also has been investigated as a material for drug delivery systems due to its chemical similarity to the inorganic phase of bones as well as its ability to adsorb and release several molecules of biological interest ${ }^{4-8}$.

The lipid bilayer of the cellular membrane acts as a barrier against permeation of various polar molecules. It also efficiently hinders the membrane permeation of nanoparticle-based therapeutics ${ }^{9,10}$. Complex formation of such therapeutic agent with endogenous peptide has strongly improved the efficiency of the delivery ${ }^{11}$, where the peptide is endowed with internalisation properties. It is named as a cell penetrating peptide (CPP). According to the literatures, CPPs could enter a variety of cells ${ }^{12}$, 13 and these peptides also enhance the cellular uptake of various macromolecular and even nanoparticulate $\operatorname{cargos}^{14-17}$. Depending on the chemical natures, different routes of cellular entry were contemplated. Most common mechanism of uptake of CPPs might be through lipid raft-dependent endocytosis, which involves either macropinocytosis $^{18}$ and caveolae pathway ${ }^{19,20}$. Some CPPs have an additional non-endocytic pathway ${ }^{21,22}$.

We have reported elsewhere ${ }^{23-26}$ that cationic polymers, such as L-lysine, L-tyrosine copolymer, poly-L-lysine, poly-L-arginine, and polyethyrenimine, could translocate across a lipid bilayer membrane ${ }^{23-25}$ and that the transporting ability of poly-L-arginine is dominated by its secondary structure ${ }^{26}$.

In the present paper we will compare physicochemical properties of the two complexes, that is, HAp nanoparticle-poly-L-arginine (abbreviated to poly(Arg)) versus HAp nanoparticle - copoly (L-arginine/ L-tryptophan $=4: 1$ ) ( abbreviated to poly(Arg,Trp). The complexes were characterized in terms of $\zeta$ potential of the HAp particle, particle size, circular dichroism (CD) and FT-IR spectra. The effect of tryptophan residues was in particular considered. The translocation through the membrane was observed by a laser scanning microscope (CLSM).

\section{EXPERIMENTAL}

poly(Arg,Trp) (L-arginine/ L-tryptophan $=4: 1$, mw 35,000$)$ and poly(Arg) $(\mathrm{mw} 35,500)$ were used 
as polypeptides. These peptides, soybean phospholipids (SBPL), HAp powder (cat. no. 677418, particle size $<200 \mathrm{~nm}$ from a BET analysis), and O-phosphorylethanolamine (opea) were purchased from Sigma-Aldrich Chemicals (St. Louis, MO, USA). All these reagents were used without further purification. Tris(hydroxymethyl)aminomethane (Tris), chloroform and ethanol were purchased from Kanto Chemical Inc. Japan. Tris-HCl buffer $(10 \mathrm{mM}, \mathrm{pH}$ 7.40) was used to adjust a solution $\mathrm{pH}$. All these reagents used here were of analytical grade.

Formation of giant liposome

SBPL $(5 \mathrm{mg})$ was dissolved in $1 \mathrm{ml}$ of chloroform in a $50 \mathrm{ml}$ round-bottomed flask. The solvent was evaporated under a gentle nitrogen stream to form a thin lipid film on the surface of the lower portion of the flask. The dried lipid film was hydrated by $10 \mathrm{ml}$ of the buffer solution, and the flask was then sealed under argon gas and incubated at $20{ }^{\circ} \mathrm{C}$ for $24 \mathrm{hr}$. The lipid film was gradually stripped off the glass surface during the incubation and formed a giant liposome suspension $^{27}$.

\section{Surface modification of HAp}

The surface of HAp was modified for CLSM measurement, of which method was mentioned elsewhere $^{26}$. To be briefly, $100 \mathrm{mg}$ of HAp nanoparticles was treated with rhodamine-labeled opea $\left(0.5 \times 10^{-4} \mathrm{~mol} / \mathrm{l}\right)$ in Tris-HCl buffer solutions (10 mM pH 7.40) at room temperature for 3 days. The HAp treated was thoroughly washed with pure water and vacuum-dried after centrifugation at room temperature for 1 day.

HAp-polypeptide complex formation

$100 \mathrm{mg}$ of HAp was dispersed in $5 \mathrm{ml}$ of $1.0 \mathrm{x}$ $10^{-6} \mathrm{~mol} / \mathrm{l}$ of polypeptide solution at room temperature. After 3 day incubation, the complex was separated from the medium by centrifugation at $3000 \mathrm{rpm}$ for $15 \mathrm{~min}$. The concentration of free polypeptide remained in a mother solution was determined by

CD-spectrophotometry.
Secondary structure change of polypeptides on the HAp was estimated by a FT-IR measurement. The sample for FT-IR observation was prepared by mixing giant liposome with HAp-polypeptide complex, which was incubated for $3 \mathrm{hr}$ at room temperature. The mixing-ratio of polymer to lipid was 1:4000 in mole. After incubation the sample was separated from an aqueous phase by ultracentrifugation at $24,000 \mathrm{rpm}$ for $30 \mathrm{~min}$ and vacuum-dried at room temperature for 1 day.

Measurements of $\zeta$ potential and particle size

$\zeta$ potentials of the particles (HAp and HAp complex) were obtained by a Nicomp 380 ZLS $\zeta$ potential/particle sizer (Santa Barbara, CA, USA). Prior to the measurement of $\zeta$ potential, mean size of the particles in the same suspension was determined. Both measurements were performed in a $1 \mathrm{~cm} \mathrm{x} 1 \mathrm{~cm}$ cuvette at a room temperature.

Measurements of circular dichroism (CD) spectra

The CD spectrum was obtained by means of a Jasco J-600 CD spectropolarimeter with a $2 \mathrm{~mm}$ quartz cuvette. The spectra were averaged over nine scans.

Measurements of FT-IR spectra

FT-IR spectrum was obtained over the range of $400-4000 \mathrm{~cm}^{-1}$ at a resolution condition of $4 \mathrm{~cm}^{-1}$ and 64 readings using a Fourier transform-infrared spectrometer (FT-IR 420, JASCO). The KBr drifts technique was used with a sample $/ \mathrm{KBr}$ ratio = $1 / 100$, where the $\mathrm{KBr}$ used was of the spectroscopic grade, Aldrich Chemical, Milwaukee, WI.

\section{CLSM observation}

A laser scanning confocal imaging system (Zeiss, LSM-410, Germany), equipped with a He-Ne laser (543 nm), was used. Samples for the CLSM observation were prepared by mixing the giant liposome with the surface modified HAp-polypeptide complex in a glass bottom dish (Matsunami Glass Ind., Japan), where the mixing ratio of polymer to lipid was 1:4000 in mole. In order to prevent photobleaching of the sample, the CLSM was operated under conservative laser

TABLE 1. $\zeta$ potential, mean diameter, and adsorption amount of peptide on the HAp particles of intact Hap, HAp-poly(Arg,Trp), and HAp-poly(Arg) complex

\begin{tabular}{ccccc}
\hline particle & $\zeta$ potential $/ \mathrm{mV}$ & mean diameter $/ \mathrm{nm}$ & adsorption amount mol/g \\
\hline intact HAp & 4.1 & $(-12.1)^{\mathrm{a}}$ & $2.0 \times 10^{2}\left(3.2 \times 10^{2}\right)^{\mathrm{a}}$ & - \\
HAp-poly(Arg,Trp) & 8.2 & $(10.6)^{\mathrm{a}}$ & $2.3 \times 10^{3}\left(1.8 \times 10^{3}\right)^{\mathrm{a}}$ & $4.5 \times 10^{-8}\left(5.1 \times 10^{-8}\right)$ \\
HAp-poly(Arg) & 25.6 & $(21.4)^{\mathrm{a}}$ & $2.3 \times 10^{3}\left(3.4 \times 10^{3}\right)^{\mathrm{a}}$ & $2.2 \times 10^{-8}\left(2.4 \times 10^{-8}\right)$ \\
\hline
\end{tabular}

${ }^{\mathrm{a}}$ Data in parentheses for $\zeta$ potential and mean diameter are those for surface modified HAp. 


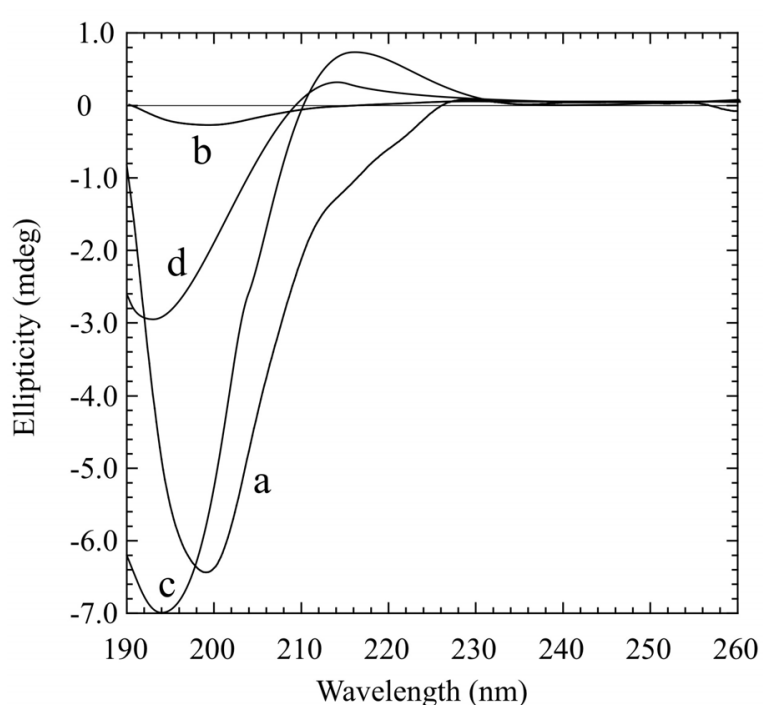

FIGURE 1. CD specra of the polypeptides $\left(1.0 \times 10^{-6}\right.$ $\mathrm{mol} / \mathrm{l}$ ) and of the supernatants of HAp-polypeptide complexes: (a) poly(Ar,Trp), (b) supernatant of the HAp-poly(Arg,Trp) complex, (c) poly(Arg) and (d) supernatant of the HAp-poly(Arg) complex.

intensity and time exposure conditions. Temperature of the observation chamber was maintained at $20{ }^{\circ} \mathrm{C}$. The images of liposomes were automatically recorded every 5 min.

\section{RESULTS}

HAp-polypeptide complexes

CD spectra of the peptides were obtained at 1.0 x $10^{-6} \mathrm{~mol} / \mathrm{L}$ poly(Arg,Trp) and poly(Arg), and also for the supernatants after centrifugation of the complexes (FIGURE 1). All CD spectra obtained showed minimum peaks below $200 \mathrm{~nm}$ of the wavelength region, which was assigned to the random coil structure ${ }^{28}$.

The $\zeta$ potential together with mean diameter of the complexes and adsorption amount of polypeptide on the HAp nanoparticle are shown in TABLE 1. The $\zeta$ potential is proportional to the surface charge density and, therefore, to the degree of binding of a cationic peptide to HAp surface. Only the surface-modified HAp particle showed a negative $\zeta$ potential value. It was caused by sulfate groups of the fluorescent dye moiety.

TABLE 1 is showing some typical data, where $\zeta$ potential shifted toward more positive value after formation of the complex. The mean diameter of HAp complexes was about ten times larger than that of the intact HAp and surface modified HAp because of aggregation after the interparticle bridging. ${ }^{29,30}$

FT-IR spectra (1800 - $1500 \mathrm{~cm}^{-1}$ region) of the intact HAp and HAp-polypeptide complexes are shown in FIGURE 2. Intact HAp exhibited no absorption band in this region, while both

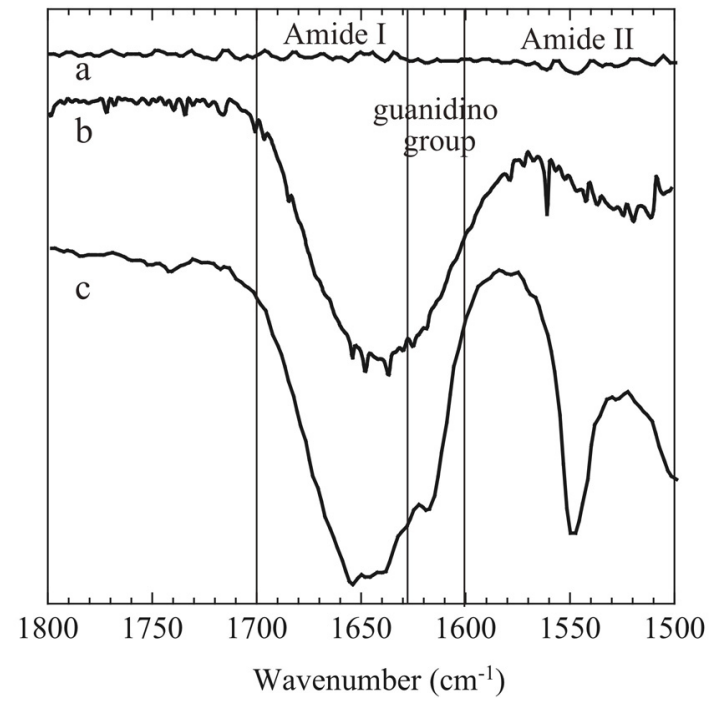

FIGURE 2. FT-IR spectra of intact Hap and HAp-polypeptide complexes: (a) intact Hap, (b) HAp-poly(Arg,Trp) complex and (c) HAp-poly(Arg) complex.

HAp-poly(Arg,Trp) and HAp-poly(Arg) complex showed the broad band around $1650 \mathrm{~cm}^{-1}$.

Liposome-complex interaction

Effect of added SBPL liposome on conformation of the polypeptide in the complex was studied by means of FT-IR spectra-(FIGURE 3a-d). The spectra of the complex in the presence of vesicle showed lipid $\mathrm{C}=\mathrm{O}$ band at $1741 \mathrm{~cm}^{-1}$ (FIGURE $3 \mathrm{~b}$ and $\mathrm{d}$ ). The vesicle exhibited a strong effect on an amide I band of the HAp-poly(Arg) complex. That is, broad band around $1650 \mathrm{~cm}^{-1}$ in the absence of vesicle changed to narrow two bands at 1618 and $1638 \mathrm{~cm}^{-1}$ in the presence of vesicle (FIGURE 3c and $\mathrm{d}$ ). On the other hand, in the case of the poly(Arg,Trp), these phenomena were not observed, as shown in FIGURE $3 a$ and $b$.

\section{CLSM imaging}

FIGURE 4 shows the CLSM images of the liposomes after addition of the surface modified HAp-poly(Arg,Trp) and surface modified HAp-poly(Arg). Fluorescence intensity of the inner aqueous phase increased $c a$. 20min after the addition of the complexes (time course not shown). It was shown that the surface modified HAp were transported through the membrane into the inner water phase. Periphery and inside of the vesicle were more bright in the system of poly(Arg) than in the system of poly(Arg,Trp), comparing FIGURE 4 $\mathrm{b}$ with a, and FIGURE $4 \mathrm{~d}$ with $\mathrm{c}$. However, the HAp-peptide complexes ( $\mu \mathrm{m}$ in size) were not detected both on the liposome membranes and in the inner aqueous phase of the liposome. It was 

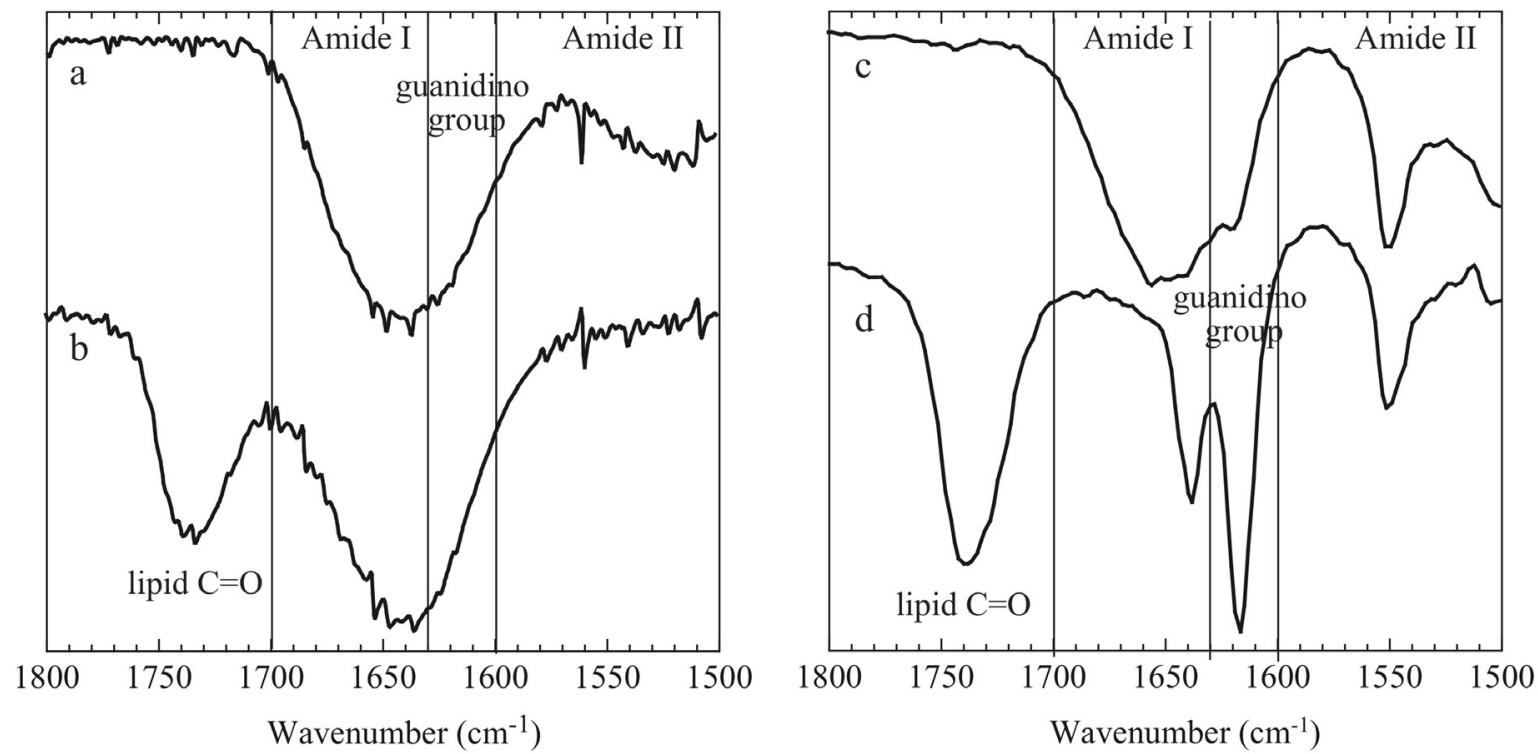

FIGURE 3. FT-IR spectra of HAp-polypeptide complexes in the presence or absence of SBPL liposome : (a) HAp-poly(Arg,Trp) complex alone, (b) HAp-poly(Arg,Trp) complex with liposome, (c) HAp-poly(Arg) complex alone, and (d) HAp-poly(Arg) complex with liposome.
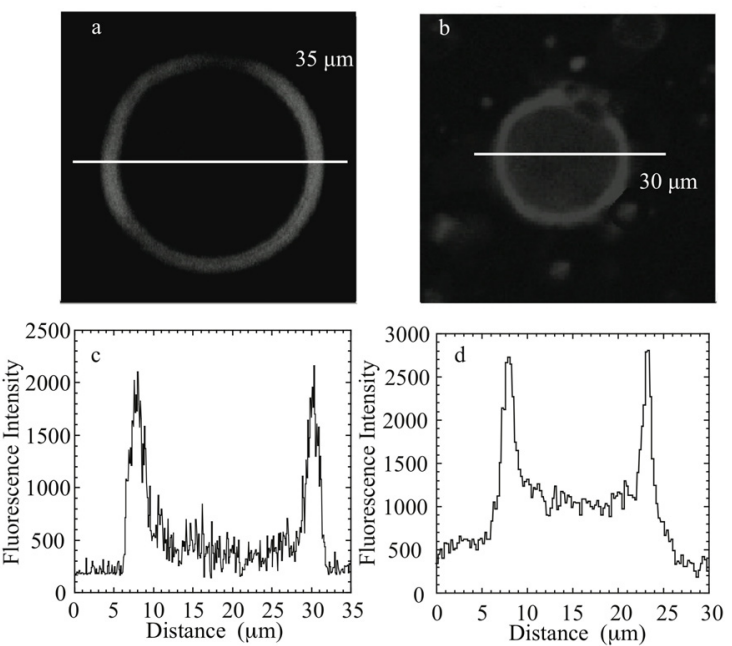

FIGURE 4. CLSM image of distribution of HAp-polypeptide complex in the presence of SBPL liposome, and fluorescence intensity profile at $20 \mathrm{~min}$ after the mixing: (a, c) for HAp-poly(Arg,Trp) complex, and $(\mathrm{b}, \mathrm{d})$ for HAp-poly(Arg) complex

Photo (a) is clearer than Photo (b). This is explained as follows; much of the dye still remains on the periphery of the vesicle in (a), while it already has diffused to the inside of the vesicle in (b). The inside of (b), therefore, is more fluorescent than that of (a). Compare also Chart (d) with Chart (c).

shown in the previous paper ${ }^{31}$ that the reduction in the size of the HAp-poly(Arg) complex during the translocation process was by virtue of breaking down of the interparticle bridges.

\section{DISCUSSION}

The fact that $\zeta$ potential shifted to more positive value in the presence of the complex(TABLE 1) suggests that both poly(Arg,Trp) and poly(Arg) are bound to the HAp and the positively charged guanidyl groups are oriented to an aqueous phase. The increased amount of $\zeta$ potential from that of the intact HAp were 4.1 and $21.5 \mathrm{mV}$ for HAp-poly(Arg,Trp) and HAp-poly(Arg), respectively. On the other hand, adsorption amount of poly(Arg,Trp) on HAp (i.e., HAp-poly(Arg,Trp) complex) was almost two times greater than that of poly(Arg) on HAp (i.e., HAp-poly(Arg) complex). These facts are suggesting that many guanidyl groups on the complex of HAp-poly(Arg,Trp) are not exposed but hidden from a water phase.

Secondary structure of poly(Arg,Trp) and poly(Arg) on the surface of the complex

The FT-IR spectra (FIGURE 2) demonstrated formation of the complex. The band between 1700 and $1640 \mathrm{~cm}^{-1}$ and around $1540 \mathrm{~cm}^{-1}$ are assigned amide I and II band ${ }^{32}$, while absorption at 1618 $\mathrm{cm}^{-1}$ is of the vibration of guanidyl groups ${ }^{29}$. The amide I band shows the conformation of polypeptide moieties are random coil. This fact is showing that the random coil structure before the binding still remained and did not change to another conformation after the binding to the HAp.

However, according to the results shown in FIGURE 3, SBPL liposome induced conformation change from random coil to $\beta$-sheet structure in poly(Arg). This transition is consistent with that reported in several papers dealing with protein structure restructuring on the surface of particles due probably to rearrangements of the hydrogen 
bonding. ${ }^{32-36}$ Secondary sructure of the poly(Arg,Trp) moieties, on the other hand, was not changed after the addition of SBPL liposome.

It was shown elsewhere ${ }^{37}$ that adsorption amount of bovine serum albumin (BSA) to the surface of HAp increased with a concentration of dodecyl trimethylammonium chloride (DTMAC) due to hydrophobic interaction between the dodecyl groups of DTMAC bound to BSA and those adsorbed on HAp at a low concentration of DTMAC, speculating formation of bilayer of BSA and DTMAC on the HAp surface. The difference between two HAp-polypeptide complexes was explained in terms of difference in adsorption structures on the HAp surface.

In other words, poly(Arg) formed adosorption monolayer on HAp by virtue of electrostaic interaction, while poly(Arg,Trp) formed adsorption bilayer; one is a layer on the HAp surface by virtue of the electrostatic interaction and the other formed on the first layer due to hydrophobic interaction between Trp residues of the peptide on the first and second layers.

Translocation through the liposome membrane

CLSM images of SBPL liposomes in the presence of HAp-polypeptide complexes (FIGURE 4) show that both complexes could translocate through the membrane. The HAp-poly(Arg) complex (FIGURE $4 \mathrm{~b}$ and $\mathrm{d}$ ) was more efficent than HAp-poly(Arg,Trp) complex (FIGURE 4a and c) in translocation.

It was shown in the previous papers ${ }^{26,24}$ that polypeptide changes its conformation when it translocates through the hydrophobic core of the lipid membrane to decrease the resistance and barrier effect. Difference in permeability between these two complexes is due mainly to the difference in flexibility of secondary structure of the polypeptide on the liposome (FIGURE 3).

\section{CONCLUSION}

We prepared HAp-poly(Arg,Trp) and HAp-poly(Arg) complexes, where the polymers were in random coil structure on HAp surface. Random incorporation of Trp residues to positively charged Arg chain induced the bilayer adsorption layer. First layer is on the HAp surface through electrostatic interaction while the second one on the first polypeptide layer after hydrophobic interaction between Trp residues. CLSM showed the complex of HAp-poly(Arg) has more flexible polypeptide structure on the HAp surface, resulting in higher ability of the membrane translocation ability. Transformation of the polypeptide secondary structure in a lipid environment is a key step of the transfer of the HAp-polypeptide complexes through the membrane. Unfortunately, however, we could not directly observe the shape of the HAp particles both at the liposome membrane and in the inner water phase by a CLSM. To our regret, it is unclear how the membrane translocation of the complex occurs and proceeds exactly with the HAp particles.

\section{ACKNOWLEDGEMENT}

This work was supported by Grant-in-Aid for Scientific Research (A) (KAKENHI 20241020).

\section{REFERENCES}

1. L. G. Ellies, J. M. Carter, J. R. Natiella, J. D. B. Featherstone, D. G. A. Nelson, J. Biomed. Mater. Res., 22, 137 (1988).

2. G. L. De Lange, C. De Putter, F. L. J. A. De Wijs, J. Biomed. Mater. Res., 24, 829 (1990).

3. R. Rossa, J. Oral. Implantol., 17, 184 (1991).

4. K. Kutsuzawa, T. Akaike, E. H. Chowdhury, Biomaterials, 29, 370 (2008).

5. M. Okazaki, Y. Yoshida, S. Yamaguchi, M. Kaneno, J.C. Elliott, Biomaterials, 22, 2459 (2001). 6. D. Wang, S. C. Miller, P. Kopečková, J. Kopeček, Adv. Drug Deliv. Rev., 57, 1049 (2005).

7. A. Barroug, M. J. Glimcher, J. Orthop. Res., 20, 274 (2002).

8. E.H. Chowdhury, A. Maruyama, A. Kano, M. Nagaoka, M. Kotaka, S. Hirose, M. Kunou, T. Akaike, Gene, 376, 87 (2006).

9. A. M. Gewirtz, D. L. Sokol, and M. Z. Ratajczak, Blood, 92, 712 (1998).

10. R. L. Juliano, S. Alahari, H. Yoo, R. Kole, M. Cho, Pharm. Res., 16, 494 (1999).

11. A. Joliot, A. Prochiantz, Adv. Drug. Deliv. Rev., 60, 608 (2008).

12. S. R. Schwarze, K. A. Hruska, and S. F. Dowdy, Trends Cell Biol., 10, 290 (2000).

13. M. A. Bogoyevitch, T. S. Kendrick, D. C. Ng and R. K. Barr, DNA Cell Biol., 21, 879 (2002).

14. H. Nagahara, A. M. Vocero-Akbani, E. L. Snyder, A. Ho, D. G. Latham, N. A. Lissy, M. Becker-HApak, S. A. Ezhevsky, S. F. Dowdy, Nat. Med. (NY), 4, 1449 (1998).

15. V. P. Torchilin, R. Rammohan, V. Weissig, T. S. Levchenko, Proc. Natl. Acad. Sci. U.S.A., 98, 8786 (2001).

16. A. Astriab-Fisher, D. Sergueev, M. Fisher, B. R. Shaw, R. L. Juliano, Pharm. Res., 19, 744 (2002).

17. U. Koppelhus, S. K. Awasthi, V. Zachar, H. U. Holst, P. Ebbesen, P. E. Nielsen, Antisense Nucleic Acid Drug Dev., 12, 51 (2002).

18. J. S. Wadia, R.V. Stan, S. F. Dowdy, Nat. Med., 10, 310 (2004).

19. A. Ferrari, V. Pellegrini, C. Arcangeli, A. Fittipaldi, M. Giacca, F. Beltram, Mol. Ther., 8, 284 (2003). 
20. A. Fittipaldi, A. Ferrari, M. Zoppe, C. Arcangeli, V. Pellegrini, F. Beltram, M. Giacca, J. Biol. Chem., 278, 34141 (2003).

21. M. E. Lindgren, M. M. Hallbrink, A. M. Elmquist, and U. Langel, Biochem. J., 377, 69 (2004).

22. S. Deshayes, A. Heitz, M. C. Morris, P. Charnet, G. Divita, and F. Heitz, Biochemistry, 43, 1449 (2004).

23. S. Liu, A. Shibata, S. Ueno, Y. Huang, Y. Wang and Y. Li, Biochem. Biophys. Res. Commun., 339, 761 (2006).

24. A. Shibata, S. Murata, S. Ueno, S. Liu, S. Futaki and Y. Baba, Biochim. Biophys. Acta, 1616, 147 (2003).

25. S. Ueno, S. Shimabayashi and A. Shibata, J. Jpn. Med. Soc. Biol. Interface, 37, 19 (2006).

26. S. Ueno, Y. Yoshioka and S, Shimabayashi, Phos. Res. Bull., 21, 71 (2007).

27. J. P. Reeves, R. M. Dowben, J. Cell Physiol., 73, 49 (1969).

28. N. Greenfield, G.D. Fasman, Biochemistry, 8, 4108 (1969).

29. T. Hayakawa, Y. Kondo, H. Yamamoto, Bull. Chem. Soc. Jpn., 42, 1937 (1969).

30. N. Brandes, P. B. Welzel, C. Werner, L.W. Kroh, J.Colloid Interface Sci., 299, 56 (2006).

31. S. Ueno, S. Shimabayashi, Phosphorus Research Bulletin, 23, 57 (2009).

32. A. Mauerer, G. Lee, Eur. J. Pharm. Biopharm., 62, 131 (2006).

33. T. Zoungrana, G.H. Findenegg, W. Norde, J.Colloid Interface Sci., 190, 437 (1997).

34. W. Norde, J.P. Favier, Colloids Surf., 64, 87 (1992).

35. P. Billsten, M. Wahlgren, T. Arnebrant, J. McGuire, H. Elwing, J. Colloid Interface Sci., 175, 77 (1995).

36. A. Kondo, J. Mihara, J. Colloid Interface Sci., 177, 214 (1996).

37. E. Motoki, S. Ueno, T. Hino, S. Shimabayashi, Phos. Res. Bull., 20, 135 (2006). 